\title{
Modulation of the Host Immune Response by Schistosome Egg-Secreted Proteins Is a Critical Avenue of Host-Parasite Communication
}

\author{
Jack P. Carson and Geoffrey N. Gobert *
}

check for

updates

Citation: Carson, J.P.; Gobert, G.N Modulation of the Host Immune

Response by Schistosome

Egg-Secreted Proteins Is a Critical

Avenue of Host-Parasite

Communication. Pathogens 2021, 10, 863. https://doi.org/10.3390/

pathogens 10070863

Academic Editor:

Krystyna Cwiklinski

Received: 25 May 2021

Accepted: 1 July 2021

Published: 8 July 2021

Publisher's Note: MDPI stays neutral with regard to jurisdictional claims in published maps and institutional affiliations.

Copyright: (c) 2021 by the authors. Licensee MDPI, Basel, Switzerland. This article is an open access article distributed under the terms and conditions of the Creative Commons Attribution (CC BY) license (https:// creativecommons.org/licenses/by/ $4.0 /)$.
School of Biological Sciences, Queen's University Belfast, 19 Chlorine Gardens, Belfast BT9 5DL, UK; jcarson22@qub.ac.uk

* Correspondence: G.Gobert@qub.ac.uk; Tel.: +44-(0)2890-972726

\begin{abstract}
During a schistosome infection, the interactions that occur between the mammalian host and the parasite change rapidly once egg laying begins. Both juvenile and adult schistosomes adapt to indefinitely avoid the host immune system. In contrast, the survival of eggs relies on quickly traversing from the host. Following the commencement of egg laying, the host immune response undergoes a shift from a type 1 helper (Th1) inflammatory response to a type 2 helper (Th2) granulomatous response. This change is driven by immunomodulatory proteins within the egg excretory/secretory products (ESPs), which interact with host cells and alter their behaviour to promote egg translocation. However, in parallel, these ESPs also provoke the development of chronic schistosomiasis pathology. Recent studies using high-throughput proteomics have begun to characterise the components of schistosome egg ESPs, particularly those of Schistosoma mansoni, S. japonicum and S. haematobium. Future application of this knowledge may lead to the identification of proteins with novel immunomodulatory activity or pathological importance. However, efforts in this area are limited by a lack of in situ or in vivo functional characterisation of these proteins. This review will highlight the current knowledge of the content and demonstrated functions of schistosome egg ESPs.
\end{abstract}

Keywords: Schistosoma mansoni; Schistosoma japonicum; Schistosoma haematobium; schistosome; egg; excretory/secretory

\section{Schistosomiasis}

Schistosomiasis affects over 200 million people worldwide. The disease is endemic primarily in the economically developing regions of Asia, South America and sub-Saharan Africa, and results in approximately 200,000 deaths annually [1,2]. Schistosoma mansoni (Africa, South America and the Middle East), S. japonicum (Southeast Asia) and S. haematobium (Africa) are the three schistosome species mainly responsible for human disease [1]. Africa carries the greatest burden of schistosomiasis, accounting for over $90 \%$ of global cases, due to the overlapping habitats of both S. mansoni and S. haematobium [3,4]. The highest level of schistosomiasis is reported in Nigeria (29 million cases), which is closely followed by the United Republic of Tanzania (19 million cases) and the Democratic Republic of Congo (15 million cases). Schistosoma japonicum is endemic in regions of China, the Philippines, and limited areas of Indonesia. In China it is estimated that less than 100,000 people are currently infected, whereas, in the Philippines, 6.7 million people are thought to live in endemic regions and 1.8 million of these are believed to be at direct risk of infection. Elimination of schistosomiasis in multiple provinces in China has recently been reported [5].

Human infection occurs when water-borne cercariae penetrate the host epidermis and transform into schistosomula. The juvenile parasites then circulate via dermal blood vessels and migrate to the intestinal mesenteric veins (S. mansoni and S. japonicum) or the venous 
plexus of the bladder (S. haematobium) [6]. Schistosomula mature into sexually distinct adult worms during this migratory phase and, upon completing their migration, form mating pairs that produce eggs, which are subsequently shed either in the faeces or urine [6]. Eggs that fail to shed from the host are instead carried away in circulation and become deposited within host tissues, commonly the liver sinusoids (S. mansoni and S. japonicum) [7] or the mucosa of the bladder wall (S. haematobium) [8]. These trapped eggs are eventually destroyed within a granuloma derived from host immune cells, however, the breakdown of a granuloma after egg death leaves behind a fibrous plaque. The accumulation of these plaques throughout long-term infection leads to reduced liver function and the development of debilitating schistosomiasis pathologies.

The pathology caused by schistosomiasis parallels changes in the parasite's lifecycle. Migrating schistosomula drive a moderate host Th1-mediated inflammatory immune response which changes to a stronger Th2-mediated immune response against parasite eggs, many of which become trapped in host tissues [7]. This Th2 response develops alongside the beginning of egg laying and is brought about by protein components released from eggs within the ESPs. These proteins modulate the responses of host immune cells, altering their cytokine and chemokine expression profiles towards a Th2 phenotype [7]. This modulation is considered to be beneficial to the traversing egg in effectively exiting from the host; studies using immunocompromised mice unable to adopt this Th1-to-Th2 shift, and thus unable to form effective granuloma, present significantly diminished levels of egg excretion in S. mansoni and S. japonicum infections [9-11]. It is not fully understood how granuloma formation impacts egg migration; however, it may act to protect the egg from inflammatory damage as it migrates through the intestinal tissues and into the lumen [12]. Furthermore, blocking this immune shift and instead maintaining a dominant Th1 response throughout chronic schistosomiasis results in significant host mortality in mouse models [13]. This is thought to occur due to unmodulated egg-related immunopathology, causing excessive inflammatory tissue damage in response to mounting egg burden [13].

Given the conflicting roles egg-secreted proteins carry out in driving the pathology associated with chronic schistosomiasis while also promoting host survival, identifying which specific proteins within the egg ESPs carry out these activities in vivo will further our understanding of the dynamics formed between host and parasite during chronic disease. Furthermore, it will also allow for the identification of novel immunomodulatory proteins that may have pharmacological relevance for the treatment of other conditions.

\section{Schistosome Egg Proteomics-Technologies and Key Studies}

The first proteomic mass spectrometry analysis of the secretome of schistosome eggs was published in 2007 when Cass and colleagues described 188 proteins within S. mansoni egg ESPs [14]. Since this original report, mass spectrometry has been used in tandem with 1- and 2-dimensional gel electrophoresis (1DE and 2DE), as well as OFFGEL electrophoresis, to further characterise the egg secretomes of S. mansoni, S. japonicum and S. haematobium. The $S$. japonicum egg secretome, investigated using $1 \mathrm{DE} /$ mass spectrometry and found to contain 95 proteins, was recently reported [15], while the S. haematobium egg secretome was found to contain 138 proteins after investigation using OFFGEL and mass spectroscopy [16]. Our own group carried out a concurrent analysis of the egg secretomes of the three main species infecting humans (S. mansoni, S. japonicum and S. haematobium) [17]. Our approach was direct, using unseparated preparations in solution, and resulted in the identification of 266, 90 and 50 proteins within the ESP profiles, respectively (Supplementary Tables S1-S3) [17]. The differences between our data for S. mansoni and those of the original study [14] were anticipated, and we attribute them to the improved reference databases and search algorithms that have become available over the intervening decade. The variation in the number of proteins detected in S. haematobium egg ESPs between our own work and that of the 2019 study by Sotillo et al. [16] is potentially due to the technical differences between the two studies. Sotillo and colleagues [16] analysed S. haematobium crude ESP using OFFGEL electrophoresis, separating their preparations into 
24 fractions which were then each subjected to mass spectroscopy analysis, contrasting with our method of using unfractionated protein preparations. The OFFGEL procedure is also considered to be more sensitive than other proteomics methods [18]. Other studies have employed similar methods to describe the secretomes of adult S. mansoni and S. japonicum worms $[19,20]$.

\subsection{Immunomodulatory S. mansoni Egg ESPs}

A summary of the distribution and function of the proteins discussed below can be seen in Table 1. As the most common experimental model for schistosomiasis, S. mansoni presents the most egg ESPs that have functional characterisation. IPSE $/ \alpha-1$, omega-1 and major egg antigen $\mathrm{P} 40$ are three of the most prominent proteins among these egg ESPs [21,22]. The hepatotoxic glycoprotein IPSE / $\alpha-1$ has been shown to bind interleukin (IL)-8, thus inhibiting the migration of neutrophils to the site of a granuloma, and to induce the degranulation of, and release of IL-4 and IL-13 from, basophils [23,24]. As well-known mediators of the Th2 response, IL-4 and IL-13 carry out essential roles in the initiation of fibrogenesis in the liver and other organs, including driving the production of collagen [25]. Omega-1, another hepatotoxic glycoprotein, possesses T2 ribonuclease activity. Omega- 1 is known to be taken up by dendritic cells (DCs), wherein it utilises its ribonuclease activity to degrade cellular rRNA and mRNA (ribosomal and messenger RNA) and influence DC function. Following exposure to omega-1, DCs have been shown to express reduced levels of IL-12, a cytokine involved in promoting the development of the Th1 response [26]. In contrast to both of these proteins, P40 has demonstrated anti-fibrotic activity and is associated with regulatory roles. In mouse models, the stimulation of lymphocytes by P40 induces both interferon (IFN) $\gamma$ and IL-2 expression, both of which promote a Th1 response [27].

Table 1. The effect(s) of immunomodulatory proteins within schistosome egg ESPs.

\begin{tabular}{|c|c|c|c|}
\hline Protein & Species & Effect(s) & Reference(s) \\
\hline \multirow{5}{*}{ IPSE $/ \alpha-1$} & \multirow{3}{*}{ S. mansoni } & Induces cell death in hepatocytes & {$[28]$} \\
\hline & & Interacts with IL-8 to inhibit neutrophil chemotaxis & [23] \\
\hline & & $\begin{array}{l}\text { Induces degranulation of basophils and subsequent release of IL- } 4 \text { and } \\
\text { IL-13 }\end{array}$ & [29] \\
\hline & \multirow[b]{2}{*}{ S. haematobium } & Localises to the nucleus of HTB-9 bladder cells & {$[30]$} \\
\hline & & $\begin{array}{l}\text { Induces angiogenesis and proliferation in EOMA endothelial cells and } \\
\text { HCV-29 urothelial cells respectively }\end{array}$ & [31] \\
\hline \multirow{2}{*}{ Omega-1 } & \multirow{2}{*}{ S. mansoni } & Induces cell death in hepatocytes & [32] \\
\hline & & Degrades dendritic cell RNA to reduce the release of IL-12 & [26] \\
\hline \multirow{3}{*}{ P40 } & S. mansoni & Induces murine lymphocytes to release IFN- $\gamma$ and IL-2 & [27] \\
\hline & \multirow{2}{*}{ S. japonicum } & Inhibits TGF- $\beta 1$-induced activation in hepatic stellate cells & [33] \\
\hline & & Induces senescence and apoptosis in hepatic stellate cells & {$[34,35]$} \\
\hline SmPEPCK & S. mansoni & $\begin{array}{l}\text { Induces proliferation and release of IFN- } \gamma \text {, IL-4 and IL- } 5 \text { from murine } \\
\text { CD } 4+\text { T cells }\end{array}$ & {$[36,37]$} \\
\hline SmVAL9 & S. mansoni & $\begin{array}{l}\text { Alters the expression profiles of MMP and TIMP enzymes in murine } \\
\text { macrophages to promote ECM degradation }\end{array}$ & [38] \\
\hline Peroxiredoxin & S. mansoni & Promotes the differentiation of alternatively activated macrophages & [39] \\
\hline Ribonuclease T2 & S. japonicum & $\begin{array}{l}\text { Inhibits dendritic cell maturation, induces macrophages to release IL-10 } \\
\text { and increases IL-4/decreases IFN- } \gamma \text { levels in the serum }\end{array}$ & [40] \\
\hline SjE16.7 & S. japonicum & Promotes neutrophil chemotaxis & [41] \\
\hline \multirow[t]{2}{*}{ Sj97 } & \multirow[t]{2}{*}{ S. japonicum } & $\begin{array}{l}\text { Interacts with complement-associated proteins C8 and C9 to inhibit } \\
\text { complement }\end{array}$ & [42] \\
\hline & & Interacts with the Fc region of human antibodies & [43] \\
\hline
\end{tabular}


Other proteins within the egg ESPs that have a proposed modulatory impact on the host immune response include SmPEPCK, SmVAL9 and peroxiredoxin. In murine models, SmPEPCK induces CD4+ T cell proliferation and primes them to release IFN- $\gamma$, IL-4 and IL-5, resulting in a mixed Th1-Th2 response [36,37]. SmVAL9 has been shown in mouse studies to induce host extracellular matrix (ECM) remodelling through the alteration of MMP (matrix metalloproteinase) and TIMP (tissue inhibitors of metalloproteinase) enzyme expression. MMPs and TIMPs are central in the regulation of the formation and resolution of fibrogenesis associated with hepatic schistosomiasis [44]. This dysregulation by SmVAL9 promotes host ECM degradation, which likely improves egg translocation through the host intestinal lumen [38]. Finally, peroxiredoxin primes a host Th2 immune response by promoting the alternative activation of macrophages (AAMs). These AAMs in turn drive the transcription of the pro-Th2 cytokines IL-4, IL-5 and IL-13 in naïve CD4+ cells [39].

\subsection{Immunomodulatory S. japonicum Egg ESPS}

S. japonicum egg ESPs contain several ribonucleases, including T2, Oy and X25, which share sequence identity with omega- 1 in $S$. mansoni ( $31 \%, 52 \%$ and $35 \%$ respectively) and its own P40 variant (66\% sequence identity with S. mansoni-P40). In murine studies, ribonuclease $\mathrm{T} 2$ stimulates a distinct Th2 immune response in the host by affecting the maturation of DCs, inducing the production of anti-inflammatory IL-10 by macrophages and the upregulation of IL-4 levels in the serum [40]. In contrast, S. japonicum-P40 inhibits fibrogenesis by reducing the activation of hepatic stellate cells and promoting apoptosis and senescence in these cells [33-35].

SjE16.7 and Sj97 are two additional significant proteins found within the egg ESPs. SjE16.7 is a strong neutrophil chemoattractant and inducer of inflammation [41], while Sj97 can inhibit components of the complement pathway and also bind directly to the Fc region of human immunoglobulins [42,43]. Sj97 has previously been associated with the cercarial and schistosomula stages in immunolocalisation studies [45]. SjE16.7 likely functions in combination with $S j$-P40 in order to regulate the developing Th2 immune response, whereas $S j 97$ is hypothesised to reduce complement-mediated cytotoxicity against juvenile and adult worms by interacting with complement-associated proteins [45]. A role of Sj97 in the egg stage is not clear, but it is possible that it has a similar protective function in reducing complement-mediated damage against eggs.

\subsection{Immunomodulatory S. haematobium Egg ESPS}

Limited research has been carried out to characterise the potential roles of S. haematobium egg-secreted proteins in the development of pathology or the modulation of the host immune response. Schistosoma haematobium eggs do, however, secrete an IPSE / $\alpha-1$ ortholog [30] with $67 \%$ sequence identity to Sm-IPSE / $\alpha-1$. Schistosoma haematobium-IPSE / $\alpha-1$ is internalised by bladder cells in culture (HTB-9), where it localises to the nucleus, indicating a potential role in regulating host cell gene expression [30]. A major consequence of chronic schistosomiasis haematobium is the possible occurrence of urogenital squamous cell carcinoma $[8,46,47]$. The potential for $S$. haematobium egg-secreted proteins to act as carcinogens has not been investigated to any great extent. However, IPSE / $\alpha-1$ is a major component of S. haematobium egg ESPs and is capable of inducing angiogenesis and cell proliferation [16,31] and, therefore, may be involved in carcinogenesis during urogenital schistosomiasis. While the functional characterisation of $S$. haematobium egg-secreted proteins is particularly lacking, comparisons can be made between these proteins and those in S. mansoni and S. japonicum egg ESPs. One example is the protein ShE16, which shares high $(70 \%)$ sequence identity with the $S$. japonicum egg ESP protein $S j E 16$, although it has not been shown if $\operatorname{ShE} 16$ is also a chemoattractant for neutrophils in a similar manner to the S. japonicum protein. 


\section{Conclusions and Future Directions}

During a schistosome infection, the commencement of egg laying marks a critical period of change to the host-parasite dynamic both in terms of the phenotype of the host immune response and the stimuli that drive pathology. While juvenile and adult schistosomes survive by avoiding the host immune response, eggs instead secrete proteins with immunomodulatory activities to manipulate the host response in a direction that promotes egg/host survival and egg transmission. Several recent studies have described the protein contents of S. mansoni, S. japonicum and S. haematobium egg ESPs; however, the function of many of these proteins remains undefined. Future research should consider characterising the in vivo role of these proteins, as doing so may lead to the identification of novel mediators of host-parasite interactions that are relevant during chronic schistosomiasis, or immunomodulatory proteins that could be repurposed as therapeutics for diseases characterised by host Th1 or Th2 responses.

The schistosome egg ESPs described here are immunomodulatory proteins that predominantly incite a host Th2 immune response and are thought to influence the Th1-to-Th2 shift that follows patency. However, few proteins that promote Th1 and mixed Th1/Th2 phenotypes have been identified, which complicate the impact that egg ESPs have on the host immune response. These proteins are expected to be involved in controlling the intensity of the developing the Th2 response that is associated with chronic schistosomiasis, giving the egg a level of control over the progression of the immune shift. As outlined here, much of the available data suggests that $S$. mansoni egg ESPs contain a greater variety of immunomodulatory components than those of S. japonicum or S. haematobium. The relatively fewer immunomodulatory proteins associated with $S$. japonicum egg secretions could represent a causative factor to the increased disease intensity this species causes in comparison to S. mansoni infections [1]. Generally, this increased pathology is credited to the greater fecundity of mature $S$. japonicum worm pairs, which results in a greater number of eggs trapped within the host tissues and an associated increase in hepatic inflammation and fibrosis [48]. However, the reduced diversity of immunomodulatory proteins within the $S$. japonicum egg ESPs could reflect an inability to effectively modulate the host immune system compared to $S$. mansoni eggs. This deficiency would result in a reduced Th1-to-Th2 shift and a more protracted chronic inflammatory state, which could produce greater pathology.

Many of the questions raised in this review could be answered by new disease models and technologies. One technology that could be used to study the functionality of specific proteins from schistosome eggs is an ex vivo model, known as the Precision Cut Liver Slice (PCLS) platform, using naïve mouse liver. PCLS replicates the cellular microenvironment of the in vivo liver and has been employed in metabolism and toxicity studies, as well as chemical and surgical fibrogenesis models [49]. The responses of mouse liver slices to Sj-SEA (S. japonicum-crude soluble egg antigen) using PCLS has been published [50], with tissue remaining unfixed, unfrozen, and alive throughout for subsequent ex vivo studies. The addition of Sj-SEA resulted in only minimal changes to overall hepatocellular morphology, and no significant increase in hepatotoxicity up to $48 \mathrm{~h}$ post-treatment. SEA concentrations were representative of those released by parasite eggs based on previous in vivo experimental infections and known liver egg burdens [51]. Going forward, this model could be used with egg ESP fractions, or individual proteins isolated from crude egg ESPs, to further clarify the role of these proteins in the in vivo setting.

Supplementary Materials: The following are available online at https:/ / www.mdpi.com/article/ 10.3390 / pathogens10070863/s1, Supplementary Table S1. Comparison of ESP data generated by Carson et al. [17] with reports from S. mansoni [14,19]. Gene Name, Accession No., Protein ID, Molecular Weight, GO ID, GO name and if the protein was reported previously in other studies, are provided. Supplementary Table S2. Comparison of ESP data generated by Carson et al. [17] with reports from S. japonicum [15,20]. Gene Name, Accession No., Protein ID, Molecular Weight, GO ID, GO name and if the protein was reported previously in other studies, are provided. Supplementary Table S3. Comparison of ESP data generated by Carson et al. [17] with reports from S. haematobium [16]. 
Gene Name, Accession No., Protein ID, Molecular Weight, GO ID, GO name and if the protein was reported previously in other studies, are provided.

Author Contributions: Conceptualization, J.P.C. and G.N.G.; writing-original draft preparation, J.P.C.; writing-review and editing, J.P.C. and G.N.G. All authors have read and agreed to the published version of the manuscript.

Funding: GNG is supported by the grant RAE FF $\backslash 1920 \backslash 1 \backslash 10$ (Royal Academy of Engineering). JPC was supported by a postgraduate studentship from the Department for the Economy (DfE) Northern Ireland.

Institutional Review Board Statement: Not applicable.

Informed Consent Statement: Not applicable.

Data Availability Statement: Data sharing not applicable.

Conflicts of Interest: The authors declare no conflict of interest.

\section{References}

1. Lewis, F.; Karunaratne, L.B.; Lewis, F.A.; Freitas, T.C.; Liang, Y. Schistosomiasis. Curr. Protoc. Immunol. $2001,19,19$.

2. Chitsulo, L.; Engels, D.; Montresor, A.; Savioli, L. The Global Status of Schistosomiasis and Its Control. Acta Trop. 2000, 77, 41-51. [CrossRef]

3. Adenowo, A.F.; Oyinloye, B.E.; Ogunyinka, B.I.; Kappo, A.P. Impact of Human Schistosomiasis in Sub-Saharan Africa. Brazilian J. Infect. Dis. 2015, 19, 196-205. [CrossRef]

4. Hailegebriel, T.; Nibret, E.; Munshea, A. Prevalence of Schistosoma Mansoni and S. Haematobium in Snail Intermediate Hosts in Africa: A Systematic Review and Meta-Analysis. J. Trop. Med. 2020, 2020, 8850840. [CrossRef]

5. Guo, J.Y.; Xu, J.; Zhang, L.J.; Lv, S.; Cao, C.L.; Li, S.Z.; Zhou, X.N. Surveillance on Schistosomiasis in Five Provincial-Level Administrative Divisions of the People's Republic of China in the Post-Elimination Era. Infect. Dis. Poverty 2020, 9, 136. [CrossRef]

6. Colley, D.G.; Bustinduy, A.L.; Secor, W.E.; King, C.H. Human Schistosomiasis. Lancet 2014, 383, 2253-2264. [CrossRef]

7. Wilson, M.S.; Mentink-Kane, M.M.; Pesce, J.T.; Ramalingam, T.R.; Thompson, R.; Wynn, T.A. Immunopathology of Schistosomiasis. Immunol. Cell Biol. 2007, 85, 148-154. [CrossRef]

8. Barsoum, R.S. Urinary Schistosomiasis: Review. J. Adv. Res. 2013, 4, 453-459. [CrossRef] [PubMed]

9. Cheever, A.W.; Poindexter, R.W.; Wynn, T.A. Egg Laying Is Delayed but Worm Fecundity Is Normal in SCID Mice Infected with Schistosoma Japonicum and S. Mansoni with or without Recombinant Tumor Necrosis Factor Alpha Treatment. Infect. Immun. 1999, 67, 2201-2208. [CrossRef] [PubMed]

10. Amiri, P.; Locksley, R.M.; Parslow, T.G.; Sadickt, M.; Rector, E.; Ritter, D.; McKerrow, J.H. Tumour Necrosis Factor $\alpha$ Restores Granulomas and Induces Parasite Egg-Laying in Schistosome-Infected SCID Mice. Nature 1992, 356, 604-607. [CrossRef] [PubMed]

11. Doenhoff, M.; Musallam, R.; Bain, J.; McGregor, A. Studies on the Host-Parasite Relationship in Schistosoma Mansoni-Infected Mice: The Immunological Dependence of Parasite Egg Excretion. Immunology 1978, 35, 771-778. [PubMed]

12. Schwartz, C.; Fallon, P.G. Schistosoma "Eggs-Iting" the Host: Granuloma Formation and Egg Excretion. Front. Immunol. 2018, 9, 2492. [CrossRef] [PubMed]

13. Rutitzky, L.I.; Hernandez, H.J.; Stadecker, M.J. Th1-Polarizing Immunization with Egg Antigens Correlates with Severe Exacerbation of Immunopathology and Death in Schistosome Infection. Proc. Natl. Acad. Sci. USA 2001, 98, 13243-13248. [CrossRef] [PubMed]

14. Cass, C.L.; Johnson, J.R.; Califf, L.L.; Xu, T.; Hernandez, H.J.; Stadecker, M.J.; Yates, J.R.; Williams, D.L. Proteomic Analysis of Schistosoma Mansoni Egg Secretions. Mol. Biochem. Parasitol. 2007, 155, 84-93. [CrossRef] [PubMed]

15. De Marco Verissimo, C.; Potriquet, J.; You, H.; McManus, D.P.; Mulvenna, J.; Jones, M.K. Qualitative and Quantitative Proteomic Analyses of Schistosoma Japonicum Eggs and Egg-Derived Secretory-Excretory Proteins. Parasit. Vectors 2019, 12, 173. [CrossRef]

16. Sotillo, J.; Pearson, M.S.; Becker, L.; Mekonnen, G.G.; Amoahid, A.S.; van Dam, G.; Corstjens, P.L.A.M.; Murray, J.; Mduluza, T.; Mutapi, F.; et al. In-Depth Proteomic Characterization of Schistosoma Haematobium: Towards the Development of New Tools for Elimination. PLoS Negl. Trop. Dis. 2019, 13, e0007362. [CrossRef]

17. Carson, J.P.; Robinson, M.W.; Hsieh, M.H.; Cody, J.; Le, L.; You, H.; McManus, D.P.; Gobert, G.N. A Comparative Proteomics Analysis of the Egg Secretions of Three Major Schistosome Species. Mol. Biochem. Parasitol. 2020, 240, 111322. [CrossRef]

18. Mulvenna, J.; Hamilton, B.; Nagaraj, S.H.; Smyth, D.; Loukas, A.; Gorman, J.J. Proteomics Analysis of the Excretory/Secretory Component of the Blood-Feeding Stage of the Hookworm, Ancylostoma Caninum. Mol. Cell. Proteom. 2009, 8, 109-121. [CrossRef]

19. Floudas, A.; Cluxton, C.D.; Fahel, J.; Khan, A.R.; Saunders, S.P.; Amu, S.; Alcami, A.; Fallon, P.G. Composition of the Schistosoma Mansoni Worm Secretome: Identification of Immune Modulatory Cyclophilin A. PLoS Negl. Trop. Dis. 2017, 11, e0006012. [CrossRef]

20. Liu, F.; Cui, S.-J.; Hu, W.; Feng, Z.; Wang, Z.-Q.; Han, Z.-G. Excretory/Secretory Proteome of the Adult Developmental Stage of Human Blood Fluke, Schistosoma Japonicum. Mol. Cell. Proteom. 2009, 8, 1236-1251. [CrossRef] 
21. Dunne, D.W.; Jones, F.M.; Doenhoff, M.J. The Purification, Characterization, Serological Activity and Hepatotoxic Properties of Two Cationic Glycoproteins (Alpha 1 and Omega 1) from Schistosoma Mansoni Eggs. Parasitology 1991, 103 Pt 2, $225-236$. [CrossRef]

22. Stadecker, M.J.; Hernandez, H.J. The Immune Response and Immunopathology in Infection with Schistosoma Mansoni: A Key Role of Major Egg Antigen Sm-P40. Parasite Immunol. 1998, 20, 217-221. [CrossRef]

23. Smith, P.; Fallon, R.E.; Mangan, N.E.; Walsh, C.M.; Saraiva, M.; Sayers, J.R.; McKenzie, A.N.J.; Alcami, A.; Fallon, P.G. Schistosoma Mansoni Secretes a Chemokine Binding Protein with Anti-Inflammatory Activity. J. Exp. Med. 2005, 202, 1319-1325. [CrossRef] [PubMed]

24. Schramm, G.; Gronow, A.; Knobloch, J.; Wippersteg, V.; Grevelding, C.G.; Galle, J.; Fuller, H.; Stanley, R.G.; Chiodini, P.L.; Haas, H.; et al. IPSE/Alpha-1: A Major Immunogenic Component Secreted from Schistosoma Mansoni Eggs. Mol. Biochem. Parasitol. 2006, 147, 9-19. [CrossRef] [PubMed]

25. Fallon, P.G.; Richardson, E.J.; McKenzie, G.J.; McKenzie, A.N.J. Schistosome Infection of Transgenic Mice Defines Distinct and Contrasting Pathogenic Roles for IL-4 and IL-13: IL-13 Is a Profibrotic Agent. J. Immunol. 2000, 164, 2585-2591. [CrossRef] [PubMed]

26. Everts, B.; Perona-Wright, G.; Smits, H.H.; Hokke, C.H.; van der Ham, A.J.; Fitzsimmons, C.M.; Doenhoff, M.J.; van der Bosch, J.; Mohrs, K.; Haas, H.; et al. Omega-1, a Glycoprotein Secreted by Schistosoma Mansoni Eggs, Drives Th2 Responses. J. Exp. Med. 2009, 206, 1673-1680. [CrossRef] [PubMed]

27. Cai, Y.; Langley, J.G.; Smith, D.I.; Boros, D.L. A Cloned Major Schistosoma Mansoni Egg Antigen with Homologies to Small Heat Shock Proteins Elicits Th1 Responsiveness. Infect. Immun. 1996, 64, 1750-1755. [CrossRef] [PubMed]

28. Abdulla, M.H.; Lim, K.C.; McKerrow, J.H.; Caffrey, C.R. Proteomic Identification of IPSE/Alpha-1 as a Major Hepatotoxin Secreted by Schistosoma Mansoni Eggs. PLoS Negl. Trop. Dis. 2011, 5, 1-10. [CrossRef]

29. Schramm, G.; Falcone, F.H.; Gronow, A.; Haisch, K.; Mamat, U.; Doenhoff, M.J.; Oliveira, G.; Galle, J.; Dahinden, C.A.; Haas, H. Molecular Characterization of an Interleukin-4-Inducing Factor from Schistosoma Mansoni Eggs. J. Biol. Chem. 2003, 278, 18384-18392. [CrossRef]

30. Pennington, L.F.; Alouffi, A.; Mbanefo, E.C.; Ray, D.; Heery, D.M.; Jardetzky, T.S.; Hsieh, M.H.; Falcone, F.H. H-IPSE Is a Pathogen Secreted Host Nucleus-Infiltrating Protein (Infiltrin) Expressed Exclusively by the Schistosoma Haematobium Egg Stage. Infect. Immun. 2017, 85, e00301-17. [CrossRef]

31. Mbanefo, E.C.; Agbo, C.T.; Zhao, Y.; Lamanna, O.K.; Thai, K.H.; Karinshak, S.E.; Khan, M.A.; Fu, C.L.; Odegaard, J.I.; Saltikova, I.V.; et al. IPSE, an Abundant Egg-Secreted Protein of the Carcinogenic Helminth Schistosoma Haematobium, Promotes Proliferation of Bladder Cancer Cells and Angiogenesis. Infect. Agent Cancer 2020, 15, 1-10. [CrossRef]

32. Dunne, D.W.; Lucas, S.; Bickle, Q.; Pearson, S.; Madgwick, L.; Bain, J.; Doenhoff, M.J. Identification and Partial Purification of an Antigen (Omega 1) from Schistosoma Mansoni Eggs Which Is Putatively Hepatotoxic in T-Cell Deprived Mice. Trans. R. Soc. Trop. Med. Hyg. 1981, 75, 54-71. [CrossRef]

33. Sun, X.; Zhang, L.; Wang, J.; Chen, J.; Zhu, D.; Shen, P.; He, X.; Pan, J.; Peng, W.; Duan, Y. Schistosoma Japonicum Protein SjP40 Inhibits TGF-B1-Induced Activation of Hepatic Stellate Cells. Parasitol. Res. 2015, 114, 4251-4257. [CrossRef] [PubMed]

34. Chen, J.; Xu, T.; Zhu, D.; Wang, J.; Huang, C.; Lyu, L.; Hu, B.; Sun, W.; Duan, Y. Egg Antigen P40 of Schistosoma Japonicum Promotes Senescence in Activated Hepatic Stellate Cells by Activation of the STAT3/P53/P21 Pathway. Cell Death Dis. 2016, 7, e2315. [CrossRef] [PubMed]

35. Chen, L.; Zhou, Q.; Liu, E.; Zhang, J.; Duan, L.; Zhu, D.; Chen, J.; Duan, Y. RSjp40 Inhibits Activated Hepatic Stellate Cells by Promoting Nuclear Translocation of YB1 and Inducing BMP-7/Smad1/5/8 Pathway. Parasit. Vectors 2019, 12, 279. [CrossRef] [PubMed]

36. Asahi, H.; Osman, A.; Cook, R.M.; LoVerde, P.T.; Stadecker, M.J. Schistosoma Mansoni Phosphoenolpyruvate Carboxykinase, a Novel Egg Antigen: Immunological Properties of the Recombinant Protein and Identification of a T-Cell Epitope. Infect. Immun. 2000, 68, 3385-3393. [CrossRef]

37. Asahi, H.; Hernandez, H.J.; Stadecker, M.J. A Novel 62-Kilodalton Egg Antigen from Schistosoma Mansoni Induces a Potent CD4+ T Helper Cell Response in the C57BL/ 6 Mouse. Infect. Immun. 1999, 67, 1729-1735. [CrossRef] [PubMed]

38. Yoshino, T.P.; Brown, M.; Wu, X.-J.; Jackson, C.J.; Ocadiz-Ruiz, R.; Chalmers, I.W.; Kolb, M.; Hokke, C.H.; Hoffmann, K.F. Excreted/Secreted Schistosoma Mansoni Venom Allergen-like 9 (SmVAL9) Modulates Host Extracellular Matrix Remodelling Gene Expression. Int. J. Parasitol. 2014, 44, 551-563. [CrossRef]

39. Donnelly, S.; Stack, C.M.; O’Neill, S.M.; Sayed, A.A.; Williams, D.L.; Dalton, J.P. Helminth 2-Cys Peroxiredoxin Drives Th2 Responses through a Mechanism Involving Alternatively Activated Macrophages. FASEB J. 2008, 22, 4022-4032. [CrossRef]

40. Ke, X.-D.; Shen, S.; Song, L.-J.; Yu, C.-X.; Kikuchi, M.; Hirayama, K.; Gao, H.; Wang, J.; Yin, X.; Yao, Y.; et al. Characterization of Schistosoma Japonicum CP1412 Protein as a Novel Member of the Ribonuclease T2 Molecule Family with Immune Regulatory Function. Parasit. Vectors 2017, 10, 89. [CrossRef]

41. Wu, C.; Chen, Q.; Fang, Y.; Wu, J.; Han, Y.; Wang, Y.; Yang, Y.; Chu, M.; Feng, Y.; Tan, L.; et al. Schistosoma Japonicum Egg Specific Protein SjE16.7 Recruits Neutrophils and Induces Inflammatory Hepatic Granuloma Initiation. PLoS Negl. Trop. Dis. 2014, 8, e2703. [CrossRef] [PubMed]

42. Deng, J.; Gold, D.; LoVerde, P.T.; Fishelson, Z. Inhibition of the Complement Membrane Attack Complex by Schistosoma Mansoni Paramyosin. Infect. Immun. 2003, 71, 6402-6410. [CrossRef] [PubMed] 
43. Loukas, A.; Jones, M.K.; King, L.T.; Brindley, P.J.; McManus, D.P. Receptor for Fc on the Surfaces of Schistosomes. Infect. Immun. 2001, 69, 3646-3651. [CrossRef] [PubMed]

44. Perry, C.R.; Burke, M.L.; Stenzel, D.J.; McManus, D.P.; Ramm, G.A.; Gobert, G.N. Differential Expression of Chemokine and Matrix Re-Modelling Genes Is Associated with Contrasting Schistosome-Induced Hepatopathology in Murine Models. PLoS Negl. Trop. Dis. 2011, 5, e1178. [CrossRef] [PubMed]

45. Gobert, G.N.; McManus, D.P. Update on Paramyosin in Parasitic Worms. Parasitol. Int. 2005, 54, 101-107. [CrossRef] [PubMed]

46. IARC Working Group on the Evaluation of Carcinogenic Risks to Humans. Biological Agents. Volume 100 B. A Review of Human Carcinogens. Int. Agency Res. Cancer 2012, 100, 1-441.

47. Ishida, K.; Hsieh, M.H. Understanding Urogenital Schistosomiasis-Related Bladder Cancer: An Update. Front. Med. 2018, 5, 223. [CrossRef]

48. Carson, J.P.; Ramm, G.A.; Robinson, M.W.; McManus, D.P.; Gobert, G.N. Schistosome-Induced Fibrotic Disease: The Role of Hepatic Stellate Cells. Trends Parasitol. 2018, 34, 524-540. [CrossRef] [PubMed]

49. Olinga, P.; Schuppan, D. Precision-Cut Liver Slices: A Tool to Model the Liver Ex Vivo. J. Hepatol. 2013, 58, 1252-1253. [CrossRef] [PubMed]

50. Gobert, G.N.; Nawaratna, S.K.; Harvie, M.; Ramm, G.A.; McManus, D.P. An Ex Vivo Model for Studying Hepatic Schistosomiasis and the Effect of Released Protein from Dying Eggs. PLoS Negl. Trop. Dis. 2015, 9, e0003760. [CrossRef]

51. Burke, M.L.; McManus, D.P.; Ramm, G.A.; Duke, M.; Li, Y.; Jones, M.K.; Gobert, G.N. Temporal Expression of Chemokines Dictates the Hepatic Inflammatory Infiltrate in a Murine Model of Schistosomiasis. PLoS Negl. Trop. Dis. 2010, 4, e598. [CrossRef] [PubMed] 